\title{
A Beam Diagnostic System for REX-ISOLDE.
}

\author{
K. Kruglov ${ }^{\text {a }}$, L. Weissman ${ }^{\mathrm{a}, 1}$, P. Van den Bergh ${ }^{\mathrm{a}}$, M. Huyse ${ }^{\mathrm{a}}$, \\ P. Van Duppen ${ }^{\text {a }}$. \\ anstituut voor Kern- en Stralingsfysica, University of Leuven, B-3001 Leuven, \\ Belgium
}

\begin{abstract}
A beam diagnostic system which can operate in wide dynamic range of beam currents and energies was developed for REX-ISOLDE. The system generates online a two dimensional beam image and its $\mathrm{x}$ and $\mathrm{y}$ projections. Features of the system and the technical design are discussed here. Numerous tests were carried out with the system to show that it satisfies the REX-ISOLDE requirements.
\end{abstract}

Key words: Beam diagnostic system; REX-ISOLDE

\section{Introduction}

The REX-ISOLDE (Radioactive Beam Experiment at ISOLDE) [1] facility, which is now under construction at CERN, aims at accelerating radioactive isotopes produced at $60 \mathrm{keV}$ at ISOLDE and to perform reaction studies that are of interest to nuclear-structure physics. Post acceleration at REX-ISOLDE consists of several stages of beam treatment. After each stage, a beam diagnostic system is required to monitor the properties of the ion beam. The position of the ion beam as well as its intensity distribution has to be monitored with a spatial resolution of $1 \mathrm{~mm}$ or better. The system must be able to operate in a wide dynamic range of currents and energies: from the $\mathrm{nA}$ region down to the level of several particles per second (pps) and from $60 \mathrm{keV}$ up to several $\mathrm{MeV} / \mathrm{amu}$ beam energies.

The majority of existing beam monitoring devices cannot satisfy all of the REX-ISOLDE requirements. Grid-based profile monitoring systems demand high currents (more then $10^{6}$ p.p.s.) and must be accompanied by expensive

$\overline{1}$ Present address: ISOLDE, CERN, EP Division, CH-1211 Geneva 23, Switzerland

Preprint submitted to Elsevier Preprint 11 July 2000 
electronics [2]. Avalanche chambers work with low currents, but require highenergy beams [3]. A. Alberts et al [4] used secondary electrons emitted from a carbon foil placed in a beam and were able to measure beams from 1 to $10^{9}$ pps. Implementation of a position sensitive electron detector allows observation of a 2-dimensional beam image if the electrons can be transported from the foil to the detector without distortion. These ideas were used in our beam diagnostic system [5] developed for REX-ISOLDE.

The diagnostic unit consists of several functional parts. A Faraday cup (FC) is used to measure currents down to the pA level. A collimator wheel driven by a step motor has several slits of different size and shape. The beam position, and intensity distribution are visualized by a beam profile monitoring system (BPMS).

As the first two devices are well known, the remainder of this article is dedicated to the BPMS and its tests.

\section{The Beam Profile Monitoring System}

The principle of the BPMS is based on secondary electrons (SEs), knocked out off an Al plate by an incoming beam [fig. 1]. Angular distribution and energy spectra of the SEs depend on beam energy and type. Fortunately, the majority of SEs have low energies (less then $10 \mathrm{eV}$ ) [6], which simplifies their manipulation. The Al plate with a grid designed to accelerate the electrons can be inserted and taken out from the beam path by means of a pneumatic feed-through. SEs are accelerated up to $5 \mathrm{keV}$ by a homogeneous electric field applied between the Al plate and the grid. From there they travel through a field free zone to the input of a microchannel plate (MCP), which is a position sensitive electron multiplier. The amplification factor of the MCP is controlled by the applied voltage and can be as high as $10^{7}$ in the chevron configuration we use. After multiplication the electrons hit a phosphor screen and produce flashes of light. This light is observed with a CCD camera, coupled to a PC. The image on the phosphor screen is stretched by a factor $\sqrt{2}$ in the horizontal direction, with respect to the beam pattern due to the fact that the beam impinges on the plate at an angle of $45^{\circ}$. The CCD camera is tilted at an angle of $45^{0}$ to the phosphor screen, which restores a 1:1 correspondence of observed image to the beam intensity pattern. The video signal from the camera is sent to the control PC where it is digitized by a frame grabber. The image of the beam is continuously updated with an update rate of 3 times per second. The software allows basic analysis of the beam intensity distribution, such as making one dimensional projections and time averaging.

Every beam diagnostic system has its own control unit. It supplies high voltage to the BPMS and controls the position of the FC, collimator wheel and $\mathrm{Al}$ 
plate. All control units are connected to the control PC via an RS485 interface. Video signals from all the BPMS are sent to the PC via a video multiplexer, while currents from the FC's are read out by a separate line via a switch card. The control PC will be integrated into the ISOLDE computer network.

\section{Dynamical Current and Energy range of the BPMS; Spatial and Timing Resolution.}

One should note that for historical reasons most of the tests were done with a microsphere plate (MSP) and CMOS camera. The MSP is very similar to the MCP device, but has a slightly worse spatial resolution.

The very first tests of the BPMS were done at the LISOL ( Leuven Isotope Separator On-Line) [7] setup in Louvain-la-Neuve (L.L.N.). A $50 \mathrm{keV}{ }^{40} \mathrm{Ar}$ beam with an intensity of several pA was clearly observed. Changes in the beam shape and intensity induced by beam optics were observed in real time [fig. 2].

The system was also tested with high-energy low intensity beams. The weakest beam which one could distinguish from the background was a 5 p.p.s $/ \mathrm{cm}^{2}$ flux of stable Xe beam with an energy of $460 \mathrm{MeV}$.

By lowering the amplification of the MCP, the BPMS was used with currents in the $\mathrm{nA}$ range.

The resolution of the system depends on the acceleration voltage, energy spectrum of the SEs and their angular distribution. The details can be found in $[5]$.

In order to measure the spatial resolution of the system, a beam collimator was built. From the whole beam it cuts out areas each of $0.3 \mathrm{~mm}$ in diameter with $1 \mathrm{~mm}$ between beam centers, creating narrow beams of approximately the same intensity close to each other. Beams of $50 \mathrm{keV} \mathrm{Ar,} 150 \mathrm{MeV}$ Ar and $30 \mathrm{MeV} \mathrm{C}$ collimated in this way gave well distinguishable spots on the screen. The result of the later experiment is shown on the fig. 3. Since the system can distinguish two beams at the distance of $1 \mathrm{~mm}$, its resolution is better than 1 mm.

Both MCP and MSP have excellent timing features. Low intensity currents (less than $10^{6} \mathrm{pps}$ ) can be measured using fast electronics by counting the individual ions.

\section{Radioactive beam tests.}

One of the major concerns for the beam diagnostic system at REX-ISOLDE was its response to radioactive background. In order to investigate such a pos- 
sibility a $50 \mathrm{keV} \alpha$-radioactive beam of ${ }^{219} \mathrm{Rn}\left(\mathrm{T}_{1 / 2}=3.96 \mathrm{~s}\right)$ was generated at LISOL in L.L.N. with an intensity of a few thousand pps. The beam was observed for several minutes by the BPMS, but no background from the radiation was seen. After stopping the beam and significant increasing amplification, one could observe a glow from the area where the beam was previously seen. When the acceleration voltage was turned off or switched to the opposite polarity, the glow disappeared. This effect appeared to be due to electrons being ripped from the Al surface by the $\alpha$-particles. Off line tests with $\alpha-\beta$ - and $\gamma$-sources mounted on the Al plate demonstrated that similar backgrounds can be produced by $\beta$ - or $\gamma$-radioactive beams as well, although, the implantation doses must be several orders of magnitude higher than for $\alpha$-radioactive beams. The mechanism for $\beta$ - or $\gamma$ induced background is similar to that of $\alpha$-radioactivity.

\section{Background from the RFQ.}

At REX-ISOLDE one of the beam diagnostic system will be positioned after the RFQ (part of the LINAC), with a magnetic quadrupole (QP) triplet between them. Strong RF field inside the RFQ generates a flux of electrons and X-rays. This flux could cause a significant background problem for the monitoring of low intensity beams .

Possible consequences of the background were studied in Munich, where the RFQ was mounted on a test beamline equipped with a plasma ion source. Without power on the magnetic lenses a strong background from the RFQ was observed. However, in the presence of magnetic field generated by the QP lenses, this background almost completely disappeared. This demonstrates that the background has two components: electrons and X-rays. The electrons do not have enough energy to pass the magnetic field. The remainder component created by X-rays is so weak that it is only visible when both the RFQ power and the MCP amplification are set to values exceeding normal working conditions.

\section{Conclusion}

A simple and reliable beam diagnostic system was developed for REX-ISOLDE. Its beam profile monitoring system generates a two-dimensional beam image with capabilities of making online $\mathrm{x}$ and y projections and time averaging. The operational principle of the beam monitoring system is based on the detection of secondary electrons knocked off the Al plate by the impinging beam. The system was tested and appeared to perform well in a wide range of beam ener- 
gies and currents ( $60 \mathrm{keV}-460 \mathrm{MeV} ; 50$ ions/s - nA). The spatial resolution of the system was found to be better than $1 \mathrm{~mm}$ and the background generated by the RFQ did not create any difficulties for the beam observation.

\section{Acknowledgements.}

We would like to express our gratitude to J. Gentens for his help in operating the LISOL separator and S. Hogg for helping to operate the Leuven Pelletron accelerator. We also wish to thank Ir. G. Berger and the Louvain-la-Neuve accelerator crew for their help in our tests with high energy beams and M. Gaelens for providing the ${ }^{7}$ Be source. We wish to thank O. Kester, T. Sieber and K. Rudolph for their support during the tests with RFQ. This work is supported by the Inter-University Attraction Poles (IUAP) Research Programm, the Fund for Scientific Research-Flandrens (FWO) and the Research Fund K.U. Leuven (GOA). M.H. is Research Director of the FWO.

\section{References}

[1] D. Habs et al., Nucl.Phys. A 616 (1997) 29-38.

[2] P. Sterhl, Rev. Sci. Instrum. 63 (4), (1992) 2652-2659.

[3] A. Breskin, G. Charpak, S. Majewski, G. Melchart, G. Petersen and F. Sauli, Nucl. Instr. and Meth. 161, (1979) 19.

[4] A. Albert, K. Kroneberger, O. Heil, K.O. Groeneveld and H. Deissel, Nucl. Instr. and Meth. A 317 (1992) 397-398.

[5] K. Kruglov, L. Weissman, P. Van den Bergh, A. Andreyev, M. Huyse, P. Van Duppen, Nucl. Instrum. and Meth. A 441 (2000) 595-604.

[6] B.L. Henke, J.P. Knauer, K. Premaratne, J. Appl. Phys 52(3) (1981) 1509.

[7] M. Huyse, P. Decrock, P. Dendooven, J. Gentens, G. Vancraynest, P. Van den Bergh and P. Van Duppen, Nucl. Instr. Meth. B 70, (1992) 50. 
Fig. 1. The Beam Profile Monitoring System. Secondary electrons produced by a beam, accelerated by an electric field, multiplied by an MCP produce light flashes on a phosphor screen.

Fig. 2. Images of the $50 \mathrm{keV}{ }^{40} \mathrm{Ar}$ beam of several $\mathrm{nA}$. The beam size, shape and intensity distribution are shown as a function of the beam optics and voltages on the BPMS.

Fig. 3. Results of the spatial resolution test using a $30 \mathrm{MeV}$ carbon beam sent via a two-hole collimator with holes diameter $0.3 \mathrm{~mm}$ and $1 \mathrm{~mm}$ between their centers. On the screen the two beam spots were clearly separated, showing a position resolution better than $1 \mathrm{~mm}$. Note also the results shown in fig. 6 of ref. [5]. Both tests were done with MSP - CMOS camera combination. 